\title{
Overexpression of chromodomain helicase DNA binding protein 5 (CHD5) inhibits cell proliferation and induces cell cycle arrest and apoptosis in chronic myeloid leukemia
}

\author{
Shilin Xiong ${ }^{1,2 \#}$, Qitao Yan ${ }^{3 \#}$, Yiqi Peng ${ }^{4}$, Sheng Huang ${ }^{5}$, Rui Zhao ${ }^{1,2}$ \\ ${ }^{1}$ Department of Biochemistry and Molecular Biology, School of Basic Medical Sciences, Southern Medical University, Guangzhou, China; \\ ${ }^{2}$ Guangdong Provincial Key Laboratory of Single Cell Technology and Application, Guangzhou, China; Department of Guangdong Provincial Key \\ Laboratory of Geriatric Infection and Organ Function Support, Geriatric Critical Care Medicine, General Hospital of Southern Theatre Command, \\ School of Computing, Guangdong University of Technology, Guangzhou, China; ${ }^{4}$ Department of Spine Surgery, Zhujiang Hospital, Southern \\ Medical University, Guangzhou, China; ${ }^{5}$ Department of Urology, Zhujiang Hospital, Southern Medical University, Guangzhou, China. \\ Contributions: (I) Conception and design: S Xiong, R Zhao; (II) Administrative support: R Zhao; (III) Provision of study materials or patients: Y Peng; \\ (IV) Collection and assembly of data: S Xiong, Q Yan; (V) Data analysis and interpretation: S Xiong, S Huang; (VI) Manuscript writing: All authors; \\ (VII) Final approval of manuscript: All authors. \\ "These authors contributed equally to this work. \\ Correspondence to: Rui Zhao. Department of Biochemistry and Molecular Biology, School of Basic Medical Sciences, Southern Medical University, \\ Guangzhou 510515, China. Email: zhaoruiruizhao@qq.com.
}

Background: Chromodomain helicase DNA binding protein 5 (CHD5) was reported to be a tumor suppressor and our previous work showed CHD5 was epigenetically inactivated in human chronic myeloid leukemia (CML). This study aimed to investigate the effect of its overexpression on CML tumorigenesis.

Methods: Quantitative reverse-transcriptase PCR and Western blotting analysis were used to detect the expression of CHD5 in human CML cell lines. The endogenous CHD5 expression was activated in two CML cell lines by CRISPR/dCas9-SAM system. In vitro cell function experiments were performed including proliferation, colony formation, apoptosis, autophagy, senescence and differentiation assays. Furthermore, tumorigenicity was evaluated in vivo in nude mice xenograft model.

Results: CHD5 was down-regulated in CML cell lines compare to normal bone marrow mononuclear cells (MCs). Cell proliferation after activating CHD5 was significantly inhibited. Moreover, overexpression of CHD5 induced G2/M phase arrest and apoptosis in CML cells. In a tumor xenograft mouse model, CHD5 restoration was found to sharply repress tumor growth. Compared with the control group, overexpression of CHD5 enhanced the expression of p21 and cdc2 phosphorylation, whereas decreased the protein level of Cyclin B1. Furthermore, experiments revealed that up-regulation of CHD5 activated caspase-3, while antiapoptosis protein Bcl-2 expression was reduced in CML cells.

Conclusions: CHD5 plays a role of anti-tumorigenic effects involved in CML cell proliferation, cell cycle arrest and apoptosis.

Keywords: Chromodomain helicase DNA binding protein 5 (CHD5); chronic myeloid leukemia (CML); cell proliferation; cell cycle arrest; apoptosis

Submitted Jun 07, 2020. Accepted for publication Dec 17, 2020.

doi: $10.21037 /$ tcr-20-2276

View this article at: http://dx.doi.org/10.21037/tcr-20-2276 


\section{Introduction}

Deletions of human chromosome $1 \mathrm{p} 36$ are common in human malignancies, and extensive investigations have focus on the search for tumor suppressor genes in this region. In 2007, chromodomain helicase DNA binding protein 5 (CHD5) was first reported as a tumor suppressor residing in human $1 \mathrm{p} 36$ in mice models with loss and gain of the region encompassing CHD5 (1) and controls cell proliferation, senescence and apoptosis via $\mathrm{p} 19^{\text {Arf }} / \mathrm{p} 53$ pathways and $\mathrm{p} 16^{\mathrm{Ink4a}} / \mathrm{Rb}$ (2). However, CHD5 is inactivated in human cancers mediated by deletions, promoter hypermethylation and microRNAs (3-5). Low CHD5 expression is strongly correlated with the development and progression of several cancers, including neuroblastoma $(6,7)$, glioma (8) and gallbladder carcinoma (9). Knockdown of CHD 5 promotes proliferation and migration in hepatocellular carcinoma (10); whereas overexpression of CHD5 suppressed proliferation and tumor growth in neuroblastoma (7) and breast cancer (11) cells. These data strongly suggest that loss of CHD5 expression could cause dysfunction of downstream signaling pathways, which might lead to tumorigenesis.

In our previous study, we found that CHD5 expression was decreased in human chronic myeloid leukemia samples compare to normal bone marrow mononuclear cells (MCs) (12), supporting its deficiency as a causal event in cancers. However, deletions of $1 \mathrm{p} 36$ were rare in chronic myeloid leukemia and did not include CHD5 (13), so expression is likely regulated by epigenetic alterations. Bisulfite DNA sequencing experiment showed that CHD5 promoter was hypermethylated at the 30 th and 31 st CpG sites in CML samples. Furthermore, the level of CHD5 expression was inversely related to the degree of methylation. CHD5 expression was up-regulated in CML cells treated with DNA methyltransferase inhibitor (12). Our previous findings indicate that repression of CHD5 in CML is regulated by promoter methylation and we thus speculate that CHD5 may play a crucial role in CML. This study aims to investigate the effect of its restoration on CML tumorigenesis and to try to explore the underlying mechanism behind it.

We confirmed that CHD5 expression was downregulated in CML cell lines, compare to MCs. The endogenous CHD5 expression was robustly activated in K562 and KBM5 cells infected with CHD5-single guide RNA (sgRNA) lentivirus. Experiments in vitro and in mice showed that activation of CHD5 markedly inhibited cell proliferation and G2 to $M$ cell-cycle transition and promoted apoptosis of CML cells. Moreover, overexpression of CHD5 up-regulated the level of p21 and cdc2 phosphorylation, and decreased levels of Cyclin B1. CHD5 restoration increased cleavage of caspase-3, while decreased Bcl-2 expression in CML cells. These results indicated that CHD5 has potent antitumor effects for CML. We present the following article in accordance with the ARRIVE reporting checklist (available at http://dx.doi. org/10.21037/tcr-20-2276).

\section{Methods}

\section{Cell culture and isolation of human bone marrow MCs}

CML cell lines KU812, K562 and KBM5 were obtained from the Culture Collection of the Chinese Academy of Sciences (Shanghai, China). Cells were maintained in RPMI-1640 medium (Gibco, Grand Island) containing $10 \%$ fetal bovine serum (FBS, Gibco, New Zealand) and $1 \%$ penicillin streptomycin (Gibco, Invitrogen, CA, USA) in an incubator at $37{ }^{\circ} \mathrm{C}$ with $5 \% \mathrm{CO}_{2}$. Healthy bone marrow samples were collected from the Southern Hospital (Guangzhou, China). MCs were isolated from bone marrow using Ficoll separation solution (GE Healthcare, Sweden).

\section{Construction of CHD5 over-expressing stable cell line}

The CRISPR/dCas9-SAM system can robustly induce endogenous gene expression of interest with the help of a target-specific guide RNA (sgRNA) and dCas9 carrying a $\mathrm{C}$-terminal tripartite synergistic activation mediator. The dCas9 lentivirus were purchased from Genechem (Shanghai, China). sgRNA precisely targeting CHD5 was design and lentivirus was constructed to effectively express CHD5 sgRNA according to standard procedures. Sequence of CHD5 sgRNA were GCCCGGGCTTTGCGGGGAGC. To obtain a cell strain for stably expressing dCas9, K562 and KBM5 were infected with the dCas9-Vp64 lentivirus (lenti) at a multiplicity of infection (MOI) of 100 with polybrene (10 $\mathrm{gg} / \mathrm{mL}$, Genechem, Shanghai, China). After 7 days, cells were grown in RPMI-1640 complete medium containing $2.5 \mu \mathrm{g} / \mathrm{mL}$ puromycin for 5 days. Positive cell lines were sub-cloned using limiting dilution and then cultured in medium containing $1 \mu \mathrm{g} / \mathrm{mL}$ puromycin for 2 weeks. The stable cell lines were expanded for the construction of 
CHD5 over-expressing stable cell line.

K562-dCas9 and KBM5-dCas9 cells were infected with lenti-CHD5-sgRNA at a MOI of 100. Cells infected with no-loadvirus were taken as negative control (NC) group. Cells were screened by G418 at 2 weeks post-infection and subsequently sub-cloned to generate stably over-expressing CHD5 cell lines. The stable cell lines were then bulk cultured for $\mathrm{s}$ the following analysis.

\section{Quantitative real-time PCR ( $q R T-P C R)$}

Total RNA was extracted with TRIzol reagent (Ambion, invitrogen, USA). The concentration and purity of RNA were measured by NaroDrop spectrometer (Thermo Fisher). RT-PCR was performed using PrimeScript ${ }^{\text {TM }}$ RT reagent Kit (Takara, Japan). The primers of CHDS gene and $\beta$-actin were designed according to published literature (12). PCR was performed with TB Green ${ }^{\mathrm{TM}}$ Premix Ex Taq $^{\text {TM }}$ II (Takara) on a ABI 7500 RTPCR system (Applied Biosystems, Thermo Fisher Scientific). Each reaction was analyzed in triplicate. The sequences of primers are as following: $\beta$-actin-R: 5 '-CTCCTTAATGTCACGCACGAT-3'; $\beta$-actin-F : 5 '-CATGTACGTTGCTATCCAGGC-3'; CHD5-R: 5'-GGTTGAGAGGAGGAAGCAGAAC-3'; CHD5-F: 5'-CGAAGGCTACAAGTATGAGCGG-3'. The relative level of $\mathrm{CHD} 5$ was calculated using the $2^{-\Delta \Delta \mathrm{Ct}}$ method.

\section{Carboxyfluorescein diacetate succinimidyl ester (CFSE) assay}

CFSE assay was used to trace cell division. Cells $\left(1 \times 10^{6}\right)$ were stained with $5 \mu \mathrm{M}$ CFSE and incubated for $20 \mathrm{~min}$ in the dark. The cells were incubated with $5 \mathrm{~mL} 1640$ medium with $10 \%$ FBS for 5 min for twice. Cells labeled with CFSE were cultured in fresh complete medium for 3 days. Cells were collected at the indicated time-point and analyzed at $488 \mathrm{~nm}$ by FACSVerse ${ }^{\mathrm{TM}}$ flow cytometer (BD Biosciences, Franklin Lakes, NJ, USA).

\section{Cell cycle distribution analysis}

Cells were collected, washed in ice-cold PBS, and fixed with $70 \%$ ethanol at $-4{ }^{\circ} \mathrm{C}$ overnight. For determination of DNA content, cells were incubated with propidium iodide (PI) and RNase A (Beyotime, China) for $30 \mathrm{~min}$ at $37^{\circ} \mathrm{C}$ in the dark. Data were collected by a flow cytometer and cell cycle distribution was analyzed by ModFit 3.0 software.

\section{Soft agar colony formation assay}

Cells $\left(5 \times 10^{3}\right)$ were mixed with $0.35 \%$ low meltingpoint agar solution in RIPM containing $10 \%$ FBS, and subsequently layered on the top of solidified agar (0.6\%). The plates were not placed into an incubator until the upper layer solidified at room temperature. Two hundred $\mu \mathrm{L}$ of complete medium was added twice weekly. After 3 weeks of incubation, colonies were fixed and stained with crystal violet. The number of colonies was counted for each plate.

\section{Senescence assay}

Cellar senescence was analyzed by senescence-associated beta-galactosidase (SA- $\beta$-gal) staining (Beyotime, China). Briefly, cells were harvested, washed with PBS 3 times and fixed in $\beta$-Galactosidase staining fixing solution for $15 \mathrm{~min}$. The fixed cells were washed twice with PBS and incubated in X-gal staining solution at $37^{\circ} \mathrm{C}$ for $12 \mathrm{~h}$. After incubation, cells were dropped on adhesion microscope slides and photographed using a light microscope at 200x (Zeiss, Germany). The percentage of SA- $\beta$-gal positive cells was obtained by counting 5 random microscope fields.

\section{Western blot}

Total protein $(50-100 \mu \mathrm{g})$ extracted RIPA lysis buffer, separated by SDS-PAGE gels and then transferred to polyvinylidene difluoride membranes. The membranes were blocked in 5\% skimmed milk (Merck KGaA, Germany) and probed using antibodies against CHD5 (1:1,000, Abcam), LC3 (1:500, CST), cleaved caspase-3 (1:1,000, CST), Bcl-2 (1:1,000, CST), p21 (1:500, CST), p53 (1:500, CST), CDK7 (1:1,000, CST), Cyclin B1 (1:2,000, CST), Cdc2 (1:1,000, CST), phospho-cdc2 (1:1,000, Tyr15, CST), tubulin $(1: 1,500$, CST $)$ and $\beta$-actin $(1: 2,000$, CST). After incubating the primary antibody at $4{ }^{\circ} \mathrm{C}$ overnight, the membranes were incubated with a HRP conjugated antibody $(1: 2,000$, Beyotime, China) for $1.5 \mathrm{~h}$. Finally, the protein bands were visualized by ECL chromogenic solution (Bio-Rad, USA).

\section{Autophagy analysis}

Transmission electron microscopy (TEM) was applied to 
localize and quantify subcellular areas of autophagy. Briefly, $1 \times 10^{6}$ cells were collected in $1.5 \mathrm{~mL} \mathrm{EP}$ tube and pelleted by centrifugation. Immediately, cell mass was fixed in $2.5 \%$ glutaraldehyde at $4{ }^{\circ} \mathrm{C}$ for $16 \mathrm{~h}$ and examined in a S-3000N transmission electron microscope at $80 \mathrm{kV}$ at Central Laboratory, Southern Medical University.

\section{Apoptosis assay}

The cell apoptosis ratio was tested with an Apoptosis Detection Kit (Beyotime, China). Cells were harvested and double-stained with annexin V and PI. Data were collected using a FACSVerse ${ }^{\mathrm{TM}}$ flow cytometer. The data was plotted using bi-exponential plots by FlowJo V10.

\section{Cell differentiation analysis}

To analyze whether CHD5 restoration has an effect on CML cell differentiation, we detected specific hematopoietic maturation markers using multi-color flow cytometry. Cells $\left(1 \times 10^{6}\right)$ were rinsed twice with flow cytometry staining buffer (Invitrogen/Thermo Fisher Scientific, USA) and stained with a cocktail containing saturating amounts of the following monoclonal antibodies: CD14-FITC (expressed on monocytes, $1 \mu \mathrm{g}$ ), CD11bPerCP-eFluor (myeloid and NK cells, $0.125 \mu \mathrm{g}$ ), CD11cAPC-eFluor (macrophages and dendritic cells, $0.5 \mu \mathrm{g}$ ) and CD66b-APC (granulocytes, $0.125 \mu \mathrm{g}$ ), CD41a-eFluor (megakaryocytes and platelets, $0.5 \mu \mathrm{g}$ ) and CD235a-PE (erythroid cells, $0.015 \mu \mathrm{g}$ ). After $30 \mathrm{~min}$ incubation at $4{ }^{\circ} \mathrm{C}$, cells were twice rinsed with flow cytometry staining buffer and were analyzed by a FACSVerse ${ }^{\mathrm{TM}}$ flow cytometer. Spectral overlap and autofluorescence were compensated single-color controls and unstained cells. The data was plotted using bi-exponential plots by FlowJo V10.

\section{Tumorigenicity assay}

The in vivo animal assays were performed as previously reported (14). Four-week-old BALB/c nude mice (half male and half female) were purchased from the Experimental Animal Center of Southern Medical University (Guangzhou, China). Male and female were randomly divided into two groups. Cells $\left(1 \times 10^{7}\right)$ were resuspended in $100 \mu \mathrm{L}$ PBS and mixed with equal volume of Matrigel (BD Biosciences, Two Oak Park, Bedford, USA) and subcutaneously injected in the right flank of the mice. Once tumors were formed, tumors were measured with calipers every 3 days for 4 weeks, and tumor volumes were measured with calipers every 4 days and calculated as below, volume $=\left(\right.$ length $\times$ width $\left.^{2}\right) / 2$. Experiments were performed under a project license [NO.: SYXK (Yue) 2016-0167] granted by the Animal Research Ethics Committee of Southern Medical University, in compliance with Southern Medical University institutional guidelines for the care and use of animals.

\section{Statistical analysis}

Data were presented as means \pm standard deviation (SD). SPSS 22.0 software (IBM, Chicago, IL, USA) was used to analyze the data. Statistical evaluation of the data was performed with Student's $t$-test. $\mathrm{P}<0.05$ (two-tailed) was considered as statistical significance.

\section{Results}

\section{CHDS expression was activated in chronic myeloid leukemia cell lines}

Our previous work showed that CHD5 was functionally silenced by promoter hypermethylation in CML samples. In this study, qRT-PCR and Western blot results showed the expression of CHD5 was significantly lower in CML cell lines (KU812, K562 and KBM5) than that in normal bone marrow MCs $(\mathrm{P}<0.01$, Figure $1 A, B, C)$. These results indicated that $\mathrm{CHD} 5$ may play a crucial role in chronic myeloid leukemia. To assess the functions of CHD5 in CML, we attempted to derive stable CHD5overexpressing CML cell lines. K562 and KBM5 had much lower expression levels of CHD5 compared to the expression in KU812 and thus were chosen to establish the CHD5-overexpressing cell lines. qRT-PCR and Western blot analysis showed CHD5 was markedly activated in lenti-CHD5-sgRNA-infected K562 and KBM5 cells, noloadvirus as $\mathrm{NC}(\mathrm{P}<0.01$, Figure 1D,E,F).

\section{Overexpression of CHDS inbibited chronic myeloid leukemia cell proliferation}

To evaluate the anti-cancer potential of CHD5, CFSE assays was applied to examine the effect of CHD5 activation on CML cell division. The results showed that CHD5 restoration dramatically reduced cellular division compared with the $\mathrm{NC}(\mathrm{P}<0.01$, Figure $2 A)$. In the soft agar colony formation assay, significantly less colonies 
A

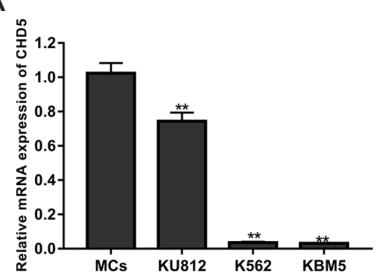

B

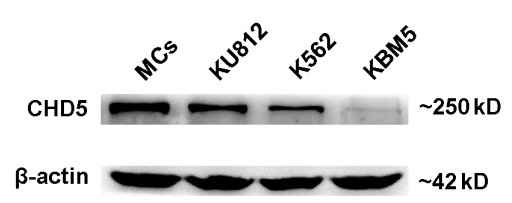

C

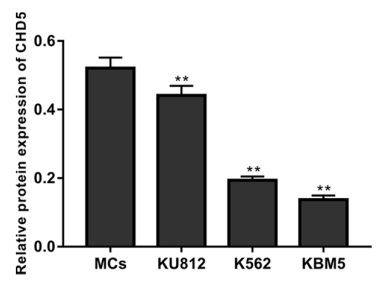

D

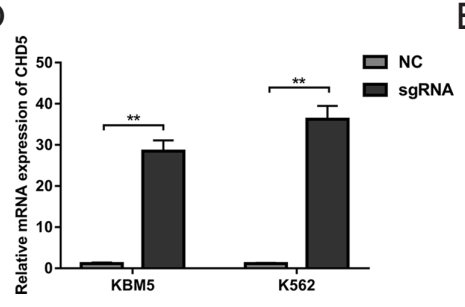

E

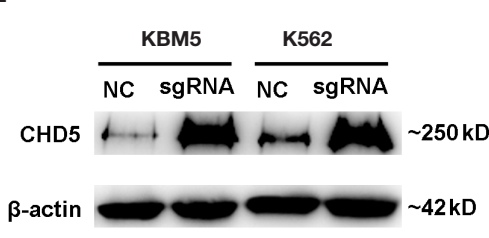

$\mathrm{F}$

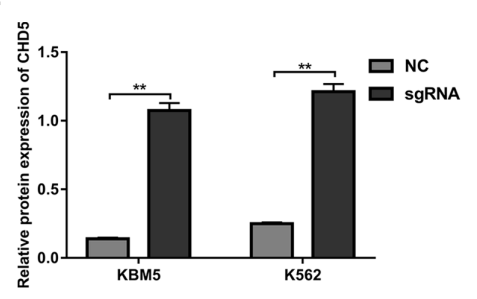

Figure 1 Activation of CHD5 expression in chronic myeloid leukemia cell lines. (A) qRT-PCR was preformed to detect mRNA level in three CML cell lines (KU812, K562 and KBM5) and normal bone marrow mononuclear cells (MCs). (B) CHD5 protein expression was tested by Western blot. (D,E) sgRNA overexpressing CHD5 at the mRNA (D) and protein (E) levels. (C,F) Protein band intensities were quantified with ImageJ and normalized to $\beta$-actin, ${ }^{* *}, \mathrm{P}<0.01$. CHD5, chromodomain helicase DNA binding protein 5; CML, chronic myeloid leukemia; sgRNA, single guide RNA.

were formed in K562-sgRNA and KBM5-sgRNA cells than in controls $(\mathrm{P}<0.01$, Figure $2 B)$. These data indicated that overexpression of CHD5 suppressed CML cell proliferation.

\section{Overexpression of CHDS induced CML cell cycle arrest at the G2/M phase arrest and apoptosis}

Since cell growth is always closely linked to the redistribution of cell cycle, we performed flow cytometry to determine CHD5 restoration would have any influence on CML cell cycle. The proportion of cells in G2/ $M$ phase was remarkably increased in sgRNA group in comparison with the control $(\mathrm{P}<0.01)$, while cell percentage in $\mathrm{S}$ phase presented a significant decrease $(\mathrm{P}<0.05$, Figure $3 A)$. To further assess the influence of CHD 5 overexpression on the biological function of CML cells, apoptosis and differentiation were analyzed by flow cytometry and SA- $\beta$-gal activity was determined using cytochemical staining method. Additionally, autophagy was examined by TEM and Western blotting. In the apoptosis assay, a distinguishable increased rate of apoptosis was observed in K562-sgRNA and KBM5sgRNA cells (Figure 3B). However, our data showed that CHD5 restoration had no impact on the cellar senescence, autophagy and differentiation $(\mathrm{P}>0.05$, Figure $\mathrm{S} 1)$. These results indicated that CHD5 overexpression induced CML cell cycle arrest and apoptosis.

\section{Overexpression of CHD 5 suppressed chronic myeloid leukemia cell growth in vivo}

In vitro experiments demonstrated that $\mathrm{CHD} 5$ inhibited cell proliferation, arrested cell cycle at G2/M phase and induced apoptosis. The anti-tumorigenesis potential of CHD5 overexpression was further tested in a BALB/c nude mouse xenograft model. The tumor volume and weight were markedly decreased in mice injected with K562-sgRNA cells, while the control cells rapidly grew into tumors $(\mathrm{P}<0.05$, Figure $4 A, B, C)$. These results indicate that $\mathrm{CHD} 5$ has potent antitumor effects in vivo.

\section{Activation of CHD5 altered the expression of cell cycle and apoptosis related proteins}

To understand the mechanism underlying CHD5-mediated anti-tumorigenicity enhancement in CML cells, we determined the effect of CHD5 on various key cell cycle regulators of $\mathrm{G} 2 / \mathrm{M}$ checkpoint, including Cyclin B1, cdc2, p53, CDK7 and p21. As shown in Figure 5A, CHD5- 
$\mathrm{A} \square \mathrm{NC}$
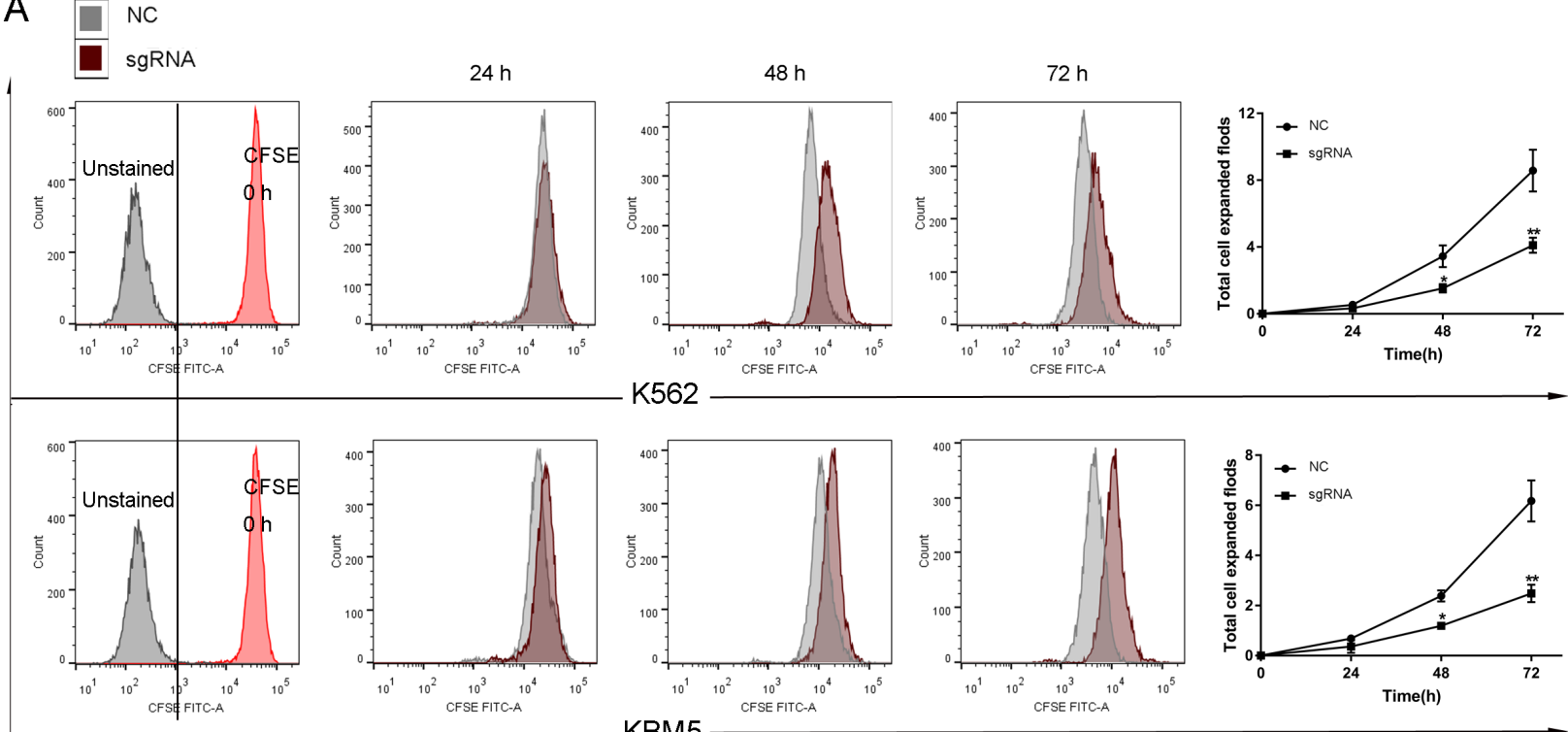
K562
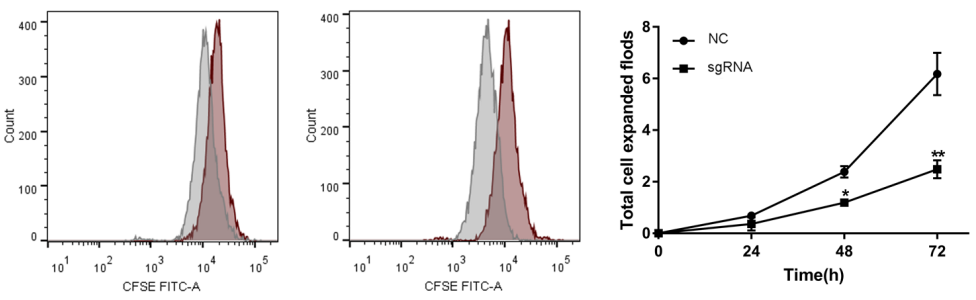

B

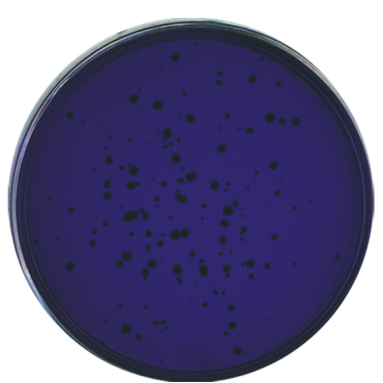

K562-NC

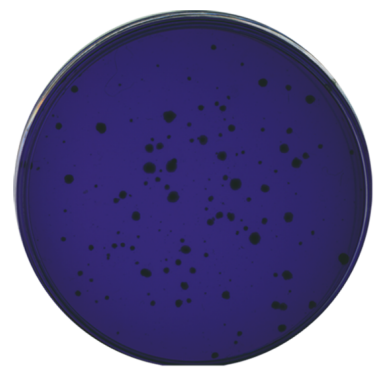

KBM5-NC

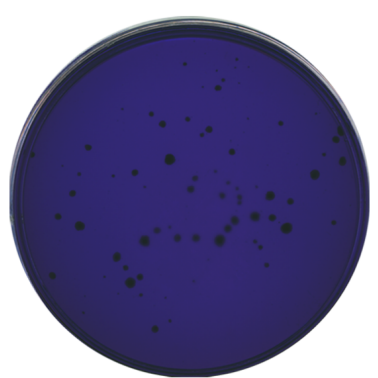

K562-sgRNA

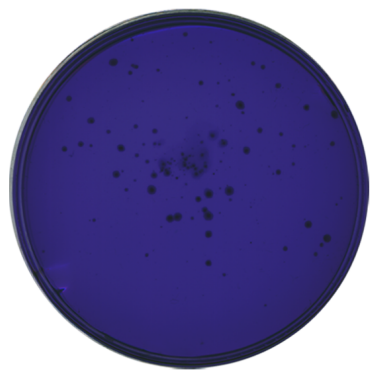

KBM5-sgRNA
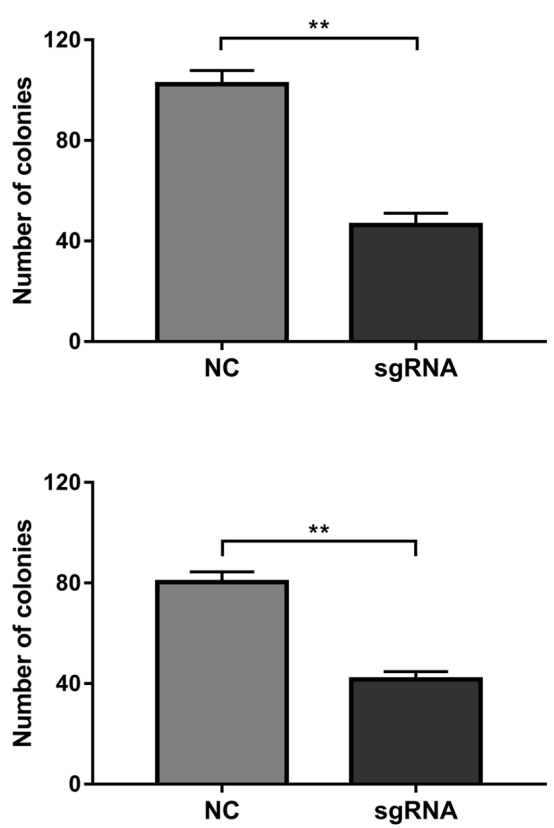

Figure 2 Activation of CHD5 inhibits chronic myeloid leukemia cell proliferation. CFSE assay (A) and soft agar colony formation assay (B) were used to determine cell proliferation. CHD5 restoration reduced cellular division and formed less colonies than in controls. ${ }^{* *}, \mathrm{P}<0.01$. CHD5, chromodomain helicase DNA binding protein 5; NC, negative control; sgRNA, single guide RNA; CFSE, carboxyfluorescein diacetate succinimidyl ester; FITC, fluorescein isothiocyanate. 

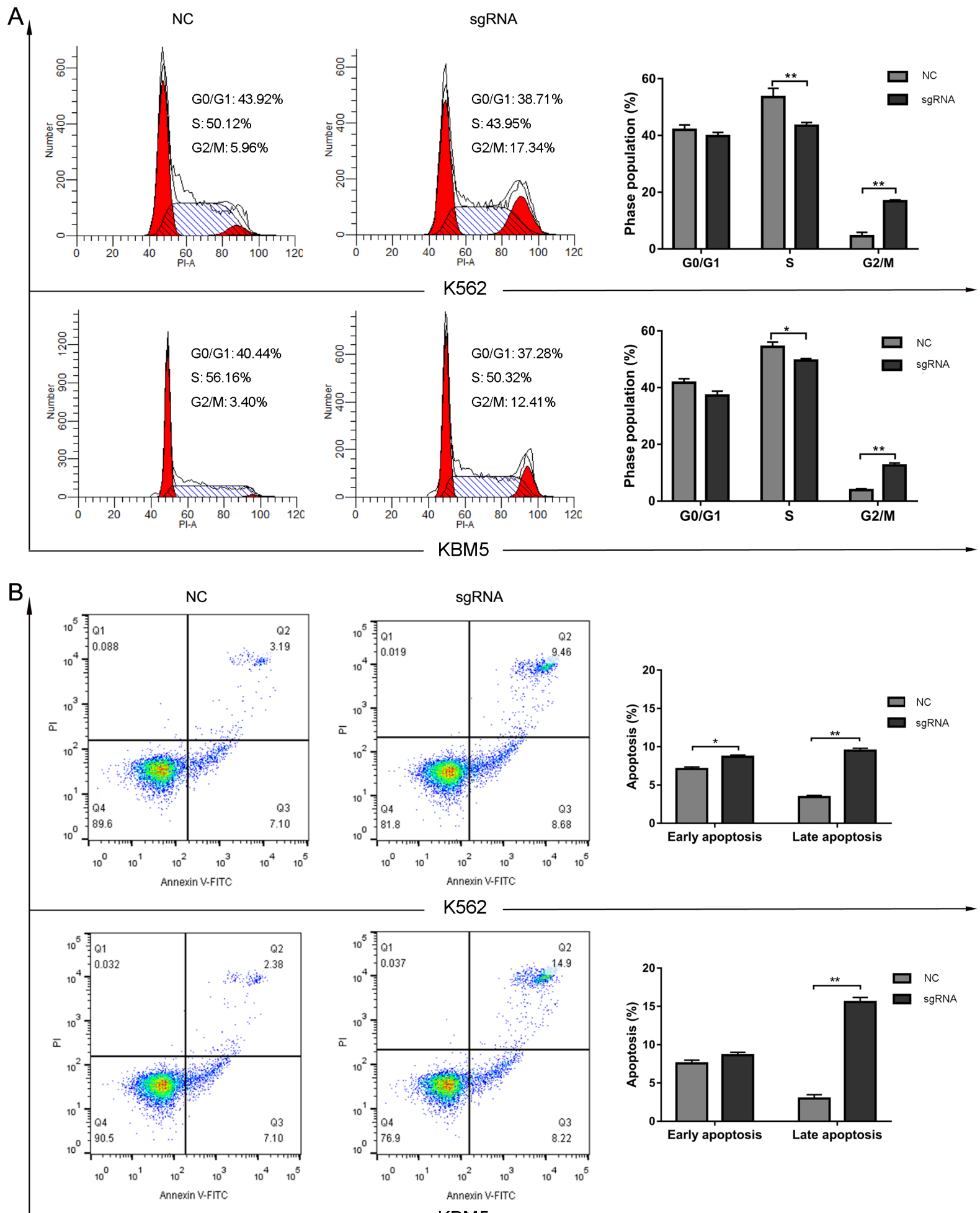

Figure 3 CHD5 restoration induced G2/M phase arrest and apoptosis. (A) Cell cycle analysis showed that cells arrested at G2/M phase notably increased in sgRNA group compared with NC group. (B) Cell apoptosis was induced by overexpression of CHD5. Statistical results are shown in the histogram, ${ }^{*}, \mathrm{P}<0.05 ;{ }^{* *}, \mathrm{P}<0.01$. CHD5, chromodomain helicase DNA binding protein 5; NC, negative control; sgRNA, single guide RNA; PI, propidium iodide; FITC, fluorescein isothiocyanate. 
A
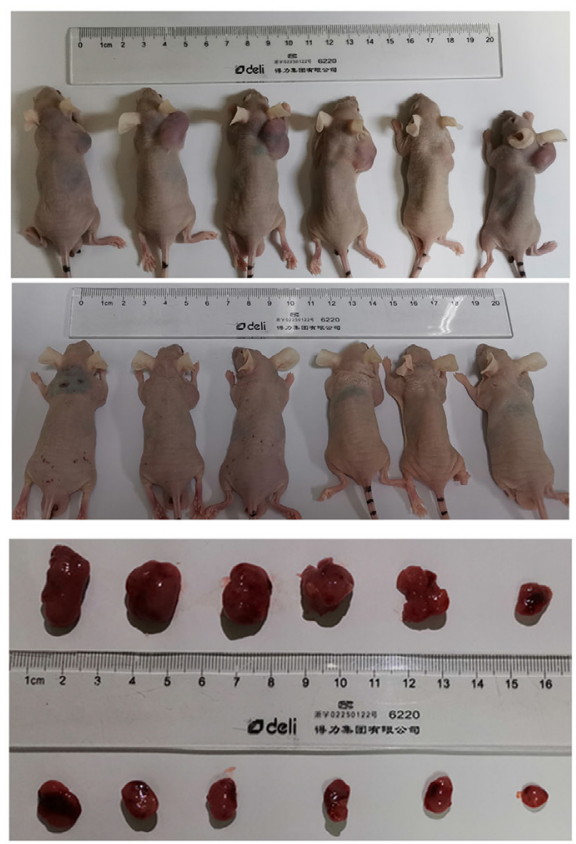

B

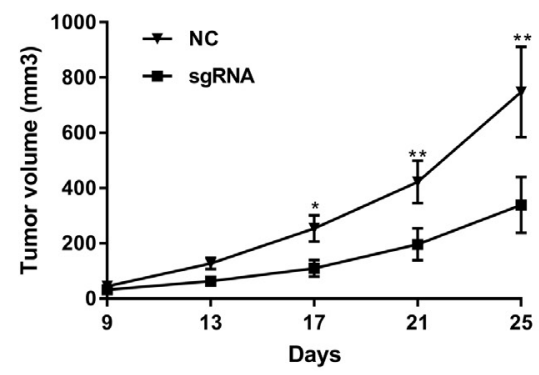

C

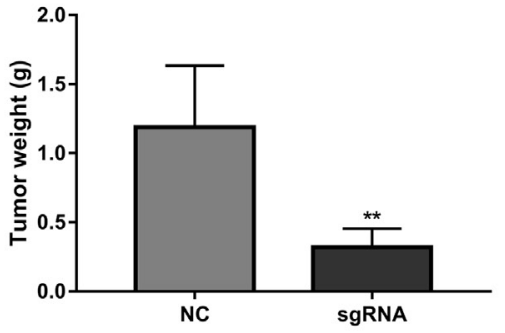

Figure 4 CHD5 overexpression impairs tumorigenesis in vivo. (A) Representative images of tumorigenicity assay performed in BALB/c nude mice. Tumor volume (B) and tumor weight (C) were significantly diminished in mice subcutaneously injected with K562-sgRNA cells, *, $\mathrm{P}<0.05$; ** $\mathrm{P}<0.01$. CHD5, chromodomain helicase DNA binding protein 5; NC, negative control; sgRNA, single guide RNA.

sgRNA substantially activated CHD5 expression in K562 and KBM5 cells. Overexpression of CHD5 suppressed the expression of Cyclin B1, enhanced the expression of cdc2 phosphorylation and p21, compared with the control cells. However, we did not observe significant changes in cdc2, p53 and CDK7 expression (Figure 5B,C). Besides, Western blot analysis showed that up-regulation of CHD5 activated caspase- 3 , while reduced the expression of anti-apoptotic protein Bcl-2 compared with controls (Figure 5D,E).

\section{Discussion}

Chronic myeloid leukemia is a common malignant proliferative disease of hematopoietic stem cells. Although the treatment of CML with tyrosine kinase inhibitors (TKIs) targeting BCR-ABL1 has evolved CML into a chronic and manageable disease $(15,16)$, drug resistance and accelerated disease progression may occur during the treatment of TKIs $(17,18)$. Therefore, further studies are needed to elucidate the molecular mechanism of CML pathogenesis and explore potential biological markers of CML.

CHD5 has been proposed as a tumor suppressor gene in several malignancies (19) and its down-regulation due to genetic and epigenetic alterations is extremely common in various types of cancer. Our previous work has identified that CHD5 is down-regulated in CML samples (12), implying that it may play an important role in the progression of CML. Concordant with the expression of CHD5 in CML samples, we detected that the expression of CHD5 was repressed in CML cell lines compared with normal bone marrow mononuclear cells in this study. These results indicated that CHD5 is inactivated in chronic myeloid leukemia. Stable CHD5-overexpressing CML cells were generated from K562 and KBM5 cell lines via CRISPR/dCas9-SAM system. CFSE and soft agar colony formation assays were used to evaluate the anti-tumour potential of CHD5 activation on CML cell proliferation. Moreover, cell cycle distribution and the proportion of apoptosis were tested by flow cytometry. The results revealed that overexpression of CHD5 suppressed cell proliferation and induced apoptosis and G2/M phase arrest. In addition, our data indicated that CHD5 restoration caused a marked reduction in the ability of CML cells to form tumors in nude mice. These results indicated that CHD5 has potent anti-tumor effect in chronic myeloid leukemia. 
A

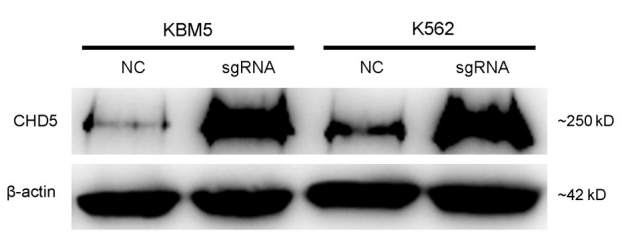

B

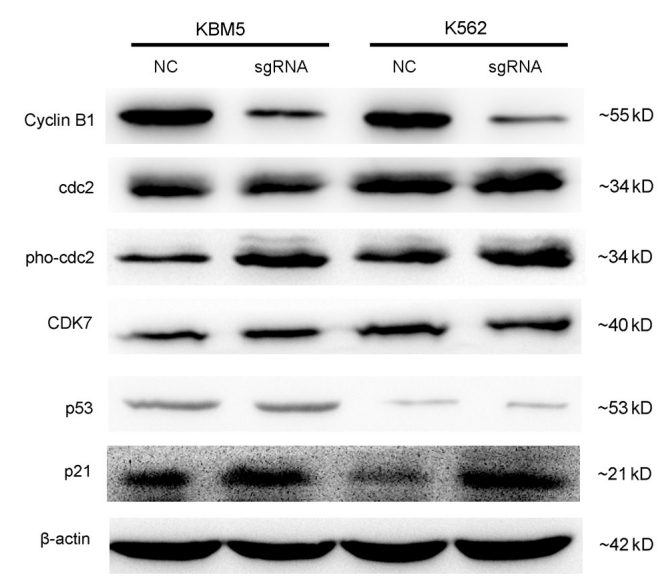

D

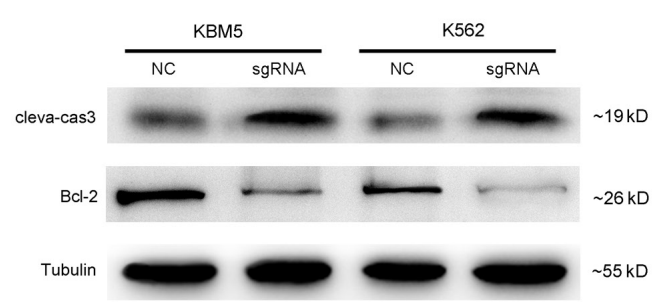

C
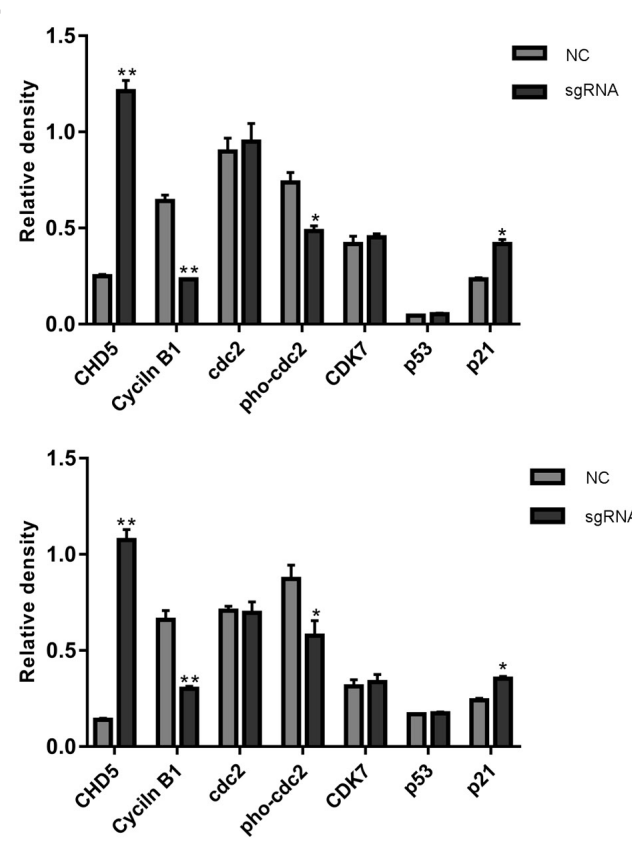

E

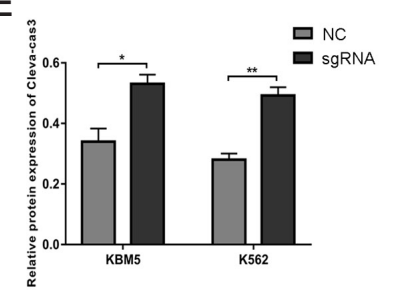

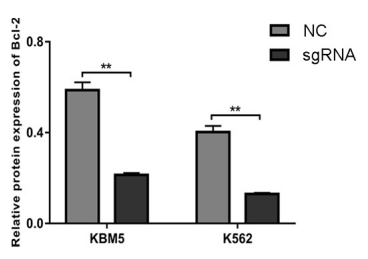

Figure 5 Alternation of cell cycle and apoptosis related proteins. (A) Effective CHD5 overexpression in K562 and KBM5 cells. (B) Western blotting analysis of Cyclin B1, cdc2, p53, CDK7 and p21. (D) The expression of cleaved caspase-3 and Bcl-2 protein. (C,E) Levels of all proteins were normalized to $\beta$-actin or tubulin, ${ }^{*}, \mathrm{P}<0.05 ;{ }^{* *}, \mathrm{P}<0.01$.

Although CHD5 has been proposed as a tumor suppressor gene, the detailed mechanism remains unclear. It has been reported in mice model that the mechanisms by which CHD5 exerts tumor suppressive effects are by activating $\mathrm{p} 19^{\mathrm{Arf}} / \mathrm{p} 53$ and $\mathrm{p} 16^{\mathrm{Ink} 4 \mathrm{a}} / \mathrm{Rb}$ pathways (2). Previous studies also reported that up-regulation of CHD5 with the CRISPR/Cas9 system decreased p53 in gastric cancer (20) and renal cell carcinoma (21). However, $p 53$ gene is inactively mutated in more than half of human cancers (22). Our study demonstrated that activation of CHD5 conferred tumor-suppressive phenotypes on K562 and KBM5 cells, though the two cell lines harbor p53 mutations, implying that there are other mechanisms for the antitumor functions of CHD5. To understand the mechanism underlying CHD5-mediated anti-tumorigenicity enhancement in CML cells, we examined the effect of CHD5 overexpression on G2/M checkpoint (Cyclin $\mathrm{B} 1-\mathrm{cdc}$ ) and other key cell cycle regulatory proteins, including $\mathrm{p} 53, \mathrm{CDK} 7$ and $\mathrm{p} 21$. The results revealed that overexpression of CHD5 down-regulated Cyclin B1 and enhanced cdc2 phosphorylation, and that the expression levels of p53 and CDK7 had no change in CML cells over-expressing CHD5. Interestingly, the expression levels of p21, a target gene of $\mathrm{p} 53$ was increased in K562sgRNA and KBM5-sgRNA cells. The results indicated that CHD5 inhibits cell proliferation, at least partially, by upregulating p53-independent p21 expression. In fact, a previous study found that CHD5 suppresses breast carcinogenesis by activating a $\mathrm{p} 53$ - and $\mathrm{Rb}$-independent p21 (11). To investigate the role of CHD5 in controlling 
apoptosis, Western blotting results revealed that $\mathrm{CHD} 5$ overexpression activated caspase- 3 and suppressed the expression level of Bcl-2.

Moreover, we also determined whether CHD5 restoration has any effects on cell senescent, autophagy and differentiation in CML cells by SA- $\beta$-gal staining method, flow cytometry, TEM and Western blotting. Our data showed that increase of CHD5 expression had no effect on cell senescence, autophagy and differentiation in K562 and KBM5 cells, though previous studies found that enhanced Chd5 could cause MEFs cellular senescence (2) and that neuroblastoma cells obtain reactivation of CHD5 expression after induction chemotherapy in high risk neuroblastoma and possess differentiation features (23), as well as treatment of neuroblastoma cells with 13-cisretinoic acid caused up-regulation of CHD5 expression and induced neuronal differentiation (24). P53 and p16 are essential for H-RASV12 induced senescence in mouse embryonic fibroblasts cells and inactivation of either $\mathrm{p} 53$ or p16 alone is sufficient to circumvent senescence (25). We thus speculate that CHD5 could not induce cell senescent, autophagy and differentiation as a result of $p 53$ mutation in K562 and KBM5 cells.

In conclusion, we demonstrated that increase of CHD5 expression suppressed CML cells proliferation and apoptosis. Overexpression of CHD5 enhanced protein expressions of p21 and cdc2 phosphorylation, whereas Cyclin B1 protein expression was decreased. Furthermore, CHD5 up-regulation activated caspase-3, while Bcl-2 expression was reduced in CML cells. This study provides clear evidence that CHD5 plays a role of anti-tumorigenic effects in the development of chronic myeloid leukemia. However, further in-depth research will be required to verify these findings and complete the apoptotic signaling pathway information.

\section{Acknowledgments}

Funding: This work was supported by the National Natural Science Foundation of China (81771710), Program of Science and Technology New Star of Zhujiang Guangzhou city, China (201610010174) and Science and Technology Program of Guangzhou, China (20170701006).

\section{Footnote}

Reporting Checklist: The authors have completed the ARRIVE reporting checklist. Available at http://dx.doi. org/10.21037/tcr-20-2276

Data Sharing Statement: Available at http://dx.doi. org/10.21037/tcr-20-2276

Conflicts of Interest: All authors have completed the ICMJE uniform disclosure form (available at http://dx.doi. org/10.21037/tcr-20-2276). All authors report grants from National Natural Science Foundation of China, grants from Program of Science and Technology New Star of Zhujiang Guangzhou city, China, grants from Science and Technology Program of Guangzhou, China, during the conduct of the study.

Ethical Statement: The authors are accountable for all aspects of the work in ensuring that questions related to the accuracy or integrity of any part of the work are appropriately investigated and resolved. Experiments were performed under a project license [NO.: SYXK (Yue) 20160167] granted by the Animal Research Ethics Committee of Southern Medical University, in compliance with Southern Medical University institutional guidelines for the care and use of animals.

Open Access Statement: This is an Open Access article distributed in accordance with the Creative Commons Attribution-NonCommercial-NoDerivs 4.0 International License (CC BY-NC-ND 4.0), which permits the noncommercial replication and distribution of the article with the strict proviso that no changes or edits are made and the original work is properly cited (including links to both the formal publication through the relevant DOI and the license). See: https://creativecommons.org/licenses/by-nc-nd/4.0/.

\section{References}

1. Bagchi A, Papazoglu C, Wu Y, et al. CHD5 is a tumor suppressor at human 1p36. Cell 2007;128:459-75.

2. Bagchi A, Mills AA. The quest for the 1 p36 tumor suppressor. Cancer Res 2008;68:2551-6.

3. Gorringe KL, Choong DY, Williams LH, et al. Mutation and methylation analysis of the chromodomain-helicaseDNA binding 5 gene in ovarian cancer. Neoplasia 2008;10:1253-8.

4. Fatemi M, Paul TA, Brodeur GM, et al. Epigenetic silencing of CHD5, a novel tumor-suppressor gene, occurs in early colorectal cancer stages. Cancer 2014;120:172-80.

5. Naraparaju K, Kolla V, Zhuang T, et al. Role of 
microRNAs in epigenetic silencing of the CHD5 tumor suppressor gene in neuroblastomas. Oncotarget 2016;7:15977-85.

6. Thompson PM, Gotoh T, Kok M, et al. CHD5, a new member of the chromodomain gene family, is preferentially expressed in the nervous system. Oncogene 2003;22:1002-11.

7. Fujita T, Igarashi J, Okawa ER, et al. CHD5, a tumor suppressor gene deleted from $1 \mathrm{p} 36.31$ in neuroblastomas. J Natl Cancer Inst 2008;100:940-9.

8. Wang L, He S, Tu Y, et al. Downregulation of chromatin remodeling factor CHD5 is associated with a poor prognosis in human glioma. J Clin Neurosci 2013;20:958-63.

9. Du X, Wu T, Lu J, et al. Decreased expression of chromodomain helicase DNA-binding protein 5 is an unfavorable prognostic marker in patients with primary gallbladder carcinoma. Clin Transl Oncol 2013;15:198204.

10. Xie CR, Li Z, Sun HG, et al. Mutual regulation between $\mathrm{CHD} 5$ and EZH2 in hepatocellular carcinoma. Oncotarget 2015;6:40940-52.

11. Wu X, Zhu Z, Li W, et al. Chromodomain helicase DNA binding protein 5 plays a tumor suppressor role in human breast cancer. Breast Cancer Res 2012;14:R73.

12. Zhao R, Meng F, Wang N, et al. Silencing of CHD5 gene by promoter methylation in leukemia. PLoS One 2014;9:e85172.

13. Mori N, Morosetti R, Spira S, et al. Chromosome band $1 \mathrm{p} 36$ contains a putative tumor suppressor gene important in the evolution of chronic myelocytic leukemia. Blood 1998;92:3405-9.

14. Huang R, Liu H, Chen Y, et al. EPS8 regulates proliferation, apoptosis and chemosensitivity in BCRABL positive cells via the BCR-ABL/PI3K/AKT/mTOR pathway. Oncol Rep 2018;39:119-28.

Cite this article as: Xiong S, Yan Q, Peng Y, Huang S, Zhao R. Overexpression of chromodomain helicase DNA binding protein 5 (CHD5) inhibits cell proliferation and induces cell cycle arrest and apoptosis in chronic myeloid leukemia. Transl Cancer Res 2021;10(2):768-778. doi: 10.21037/tcr-20-2276
15. Druker BJ, Guilhot F, O'Brien SG, et al. Five-year followup of patients receiving imatinib for chronic myeloid leukemia. N Engl J Med 2006;355:2408-17.

16. Kantarjian H, Cortes JE. Complete cytogenetic response, not deep molecular response, is associated with survival in chronic myeloid leukemia. J Clin Oncol 2014;32:3077.

17. Lee CR, Kang JA, Kim HE, et al. Secretion of IL-1beta from imatinib-resistant chronic myeloid leukemia cells contributes to BCR-ABL mutation-independent imatinib resistance. FEBS Lett 2016;590:358-68.

18. Mughal T, Cortes J, Cross NC, et al. Chronic myeloid leukemia--some topical issues. Leukemia 2007;21:1347-52.

19. Baykara O, Tansarikaya M, Bulut P, et al. CHD5 is a potential tumor suppressor in non small cell lung cancer (NSCLC). Gene 2017;618:65-8.

20. Hashimoto T, Kurokawa Y, Wada N, et al. Clinical significance of chromatin remodeling factor CHD5 expression in gastric cancer. Oncol Lett 2020;19:1066-73.

21. Du Z, Li L, Huang X, et al. The epigenetic modifier CHD5 functions as a novel tumor suppressor for renal cell carcinoma and is predominantly inactivated by promoter CpG methylation. Oncotarget 2016;7:21618-30.

22. Hollstein M, Shomer B, Greenblatt M, et al. Somatic point mutations in the p53 gene of human tumors and cell lines: updated compilation. Nucleic Acids Res 1996;24:141-6.

23. Garcia I, Mayol G, Rodriguez E, et al. Expression of the neuron-specific protein CHD5 is an independent marker of outcome in neuroblastoma. Mol Cancer 2010;9:277.

24. Higashi M, Kolla V, Iyer R, et al. Retinoic acid-induced CHD5 upregulation and neuronal differentiation of neuroblastoma. Mol Cancer 2015;14:150.

25. Serrano M, Lin AW, McCurrach ME, et al. Oncogenic ras provokes premature cell senescence associated with accumulation of p53 and p16INK4a. Cell 1997;88:593-602. 
A

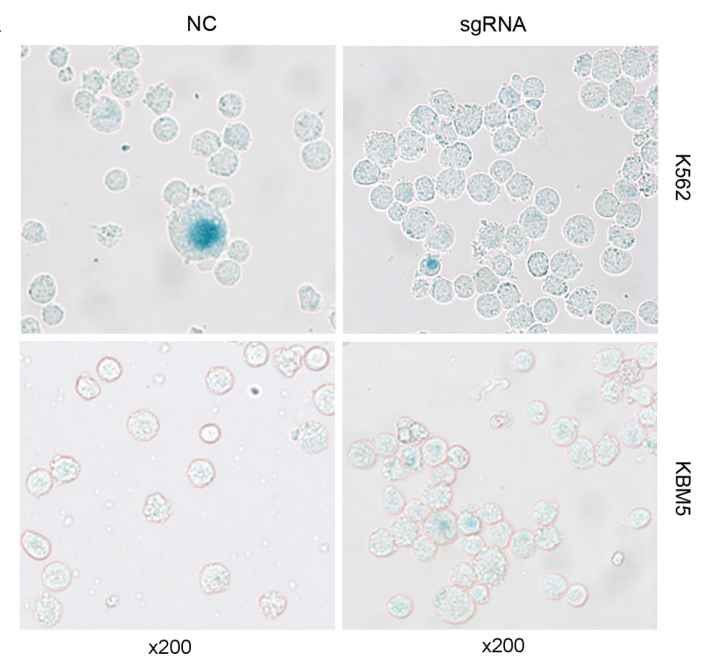

B
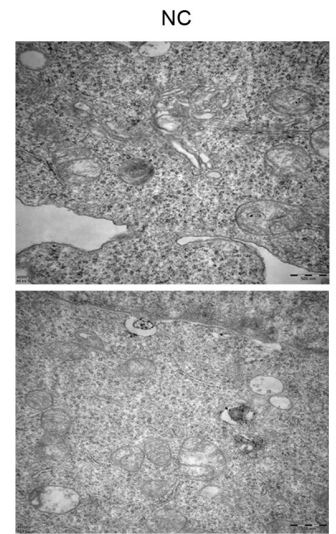

D
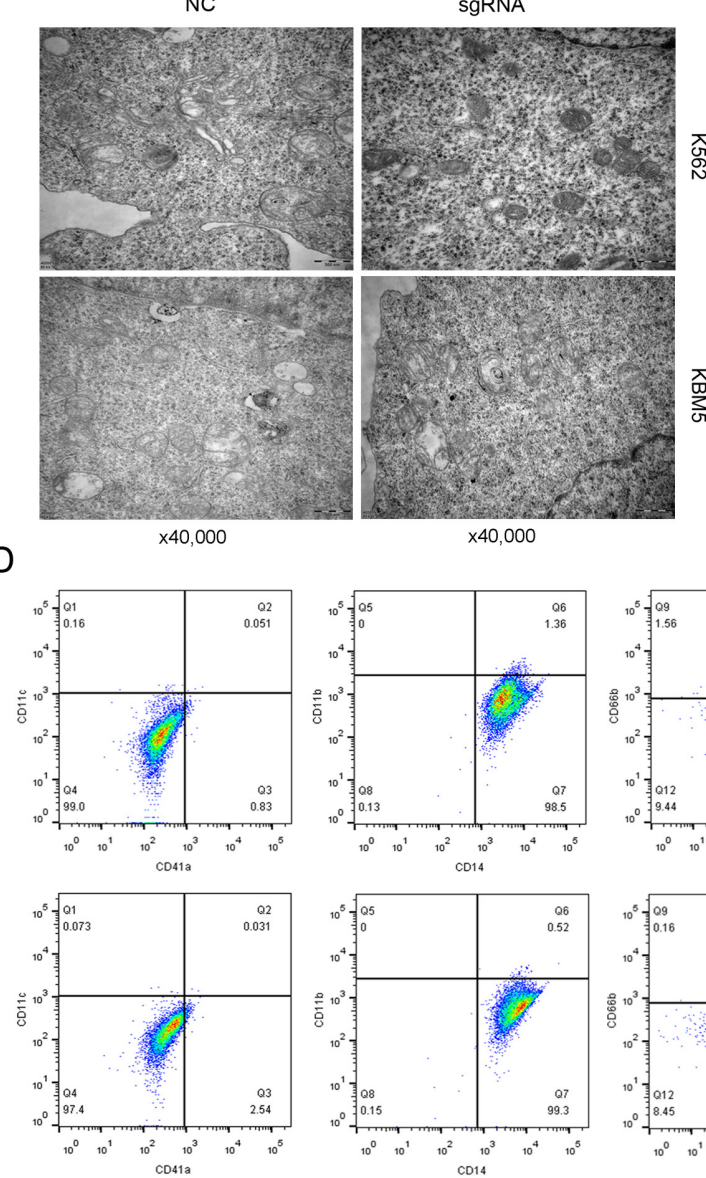

쟁

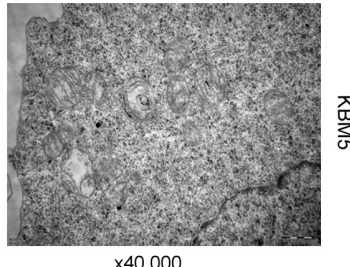

否

$\times 40,000$
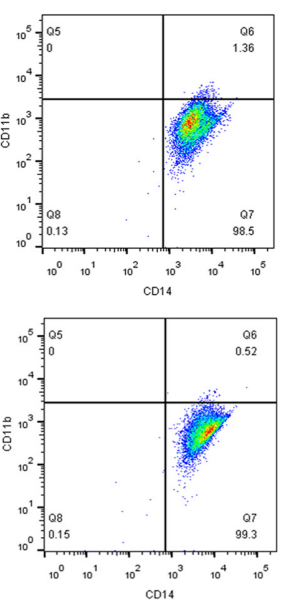

정
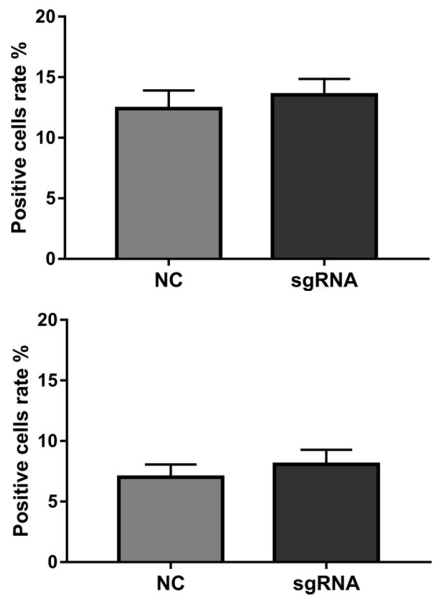

C
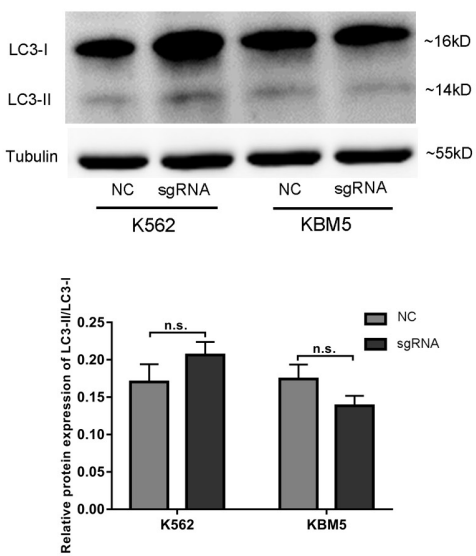
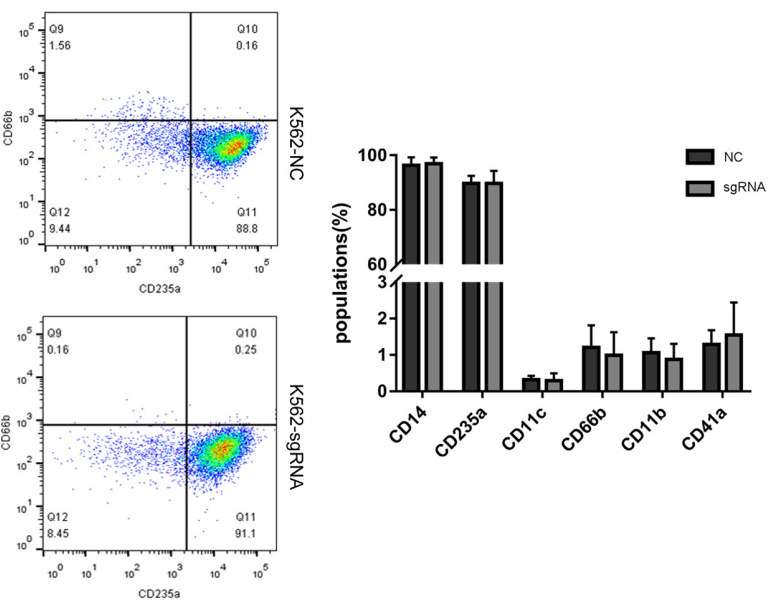

Figure S1 Overexpression of CHD5 has no effects on cell senescent, autophagy and differentiation. (A) Senescence-associated betagalactosidase (SA- $\beta$-gal) activity (200x). (B) Transmission electron microscopy analysis was performed to localize and quantify subcellular areas of autophagy. (C) Western blot analysis of the levels of LC3-I and LC3-II protein. (D) Cell differentiation features analyzed by fluorescence activated cell sorting. CHD5, chromodomain helicase DNA binding protein 5; NC, negative control; sgRNA, single guide RNA. 